\title{
Xavier Zubiri y la creación de Dios*
}

\author{
Hugo Gudiel, S.J., \\ Centro de Reflexión Teológica
}

\section{Introducción}

Con el tema de la creación de Dios, desde la perspectiva del problema teologal del hombre en Xavier Zubiri, continuamos presentando, de manera muy general en este artículo, los "conceptos fundamentales que constituyen el contenido del cristianismo". Anteriormente, vimos cómo el autor entiende y conceptúa la realidad trinitaria de Dios (PTHC 149) ${ }^{1}$. Como mostraremos a lo largo de la exposición, esa concepción trinitaria de la realidad de Dios es la que precisamente se pone de manifiesto en toda la temática de la realidad de la creación de las cosas y, de modo muy particular, en la creación de la realidad del hombre.

En este artículo pretendo exponer, de modo descriptivo, la realidad de la creación divina tal como aparece en el capítulo III de la obra de Zubiri, El problema teologal del hombre: Cristianismo. Varias son las cuestiones fundamentales que se desprenden de este trabajo: ¿Qué significado tiene la creación? ¿Cuál es el carácter formal y la estructura misma del acto creador? ¿Cuáles son los distintos modos de creación por parte de Dios? ¿Qué lugar ocupa la realidad humana en el contexto de la creación trinitaria de Dios? ¿Puede deducirse alguna contribución de Zubiri a la antropología teológica trinitaria a partir de este trabajo? Son estas preguntas básicas que emergen y se sugieren a partir del mismo trabajo de Zubiri, y que pueden contribuir a orientar mejor su exposición y lectura.

* El texto que a continuación presentamos procede de la tercera parte del curso de 1971 que Zubiri dictó sobre el problema teologal del hombre, y que ha sido recogido como "Capítulo III: Creación", en su obra El problema teologal del hombre: Cristianismo, pp. 149-231. En este trabajo pondremos las notas de esta obra con la sigla PTHC y su/s respectiva/s página/s.

1. Sobre ese tema, véase X. Zubiri, El problema teologal del hombre: Cristianismo, en el "Capítulo II: Trinidad", pp. 87-147. También H. C. Gudiel García, "La revelación del misterio de la Trinidad en Xavier Zubiri”, Revista Latinoamericana de Teología 81 (2010), pp. 343-373. 
Por la vía de la transcendencia, Dios no solo transciende al mundo, sino que también el mundo es transcendente a Dios. A esto segundo es a lo que de una manera muy general, y que habrá que explicar a continuación, aquí se llama creación. Dos son las grandes secciones que se desarrollan en este trabajo. En la primera se responde a la pregunta qué es la creación y, por tanto, es el momento en el que Zubiri nos proporciona su concepción de la creación. Y en la segunda sección se abordan los distintos modos de creación por parte de Dios, destacándose en modo particular la creación de la realidad humana y su carácter intrínsecamente trinitario (cfr. PTHC 151)2.

\section{Qué es la creación de Dios}

Toda realidad, por el hecho de ser real y por su propio carácter de realidad, es activa en sí misma y por sí misma. De ahí que la actividad se identifique con la misma realidad. En consecuencia, la realidad es formal y constitutivamente un "dar de sî". En el caso de Dios, ese dar de sí se expresa en forma de "procesiones trinitarias". Pero en la creación también acontece un riguroso dar de sí. Ahí Dios da de sí la realidad otra que es precisamente el mundo (PTHC 152).

Ahora bien, este dar de sí no solo significa que Dios sea el "hacedor del mundo", porque ciertamente el mundo puede hacerse de muchas maneras. Veremos a continuación cómo incluso en el Antiguo Testamento el relato yahvista de la creación ( $c f r$. Gn 2, 4b y ss.) está fundado justo en esta idea del hacer: "hizo Yahveh Dios" (Gn 2, 4). Pero este concepto de creación es insuficiente para Zubiri porque, desde su perspectiva, "Dios es más que hacedor del mundo" (PTHC 152).

La creación, pues, no es un simple hacer. Pero tampoco puede admitirse la idea en virtud de la cual la creación es una "emanación": según esta, Dios tira de sí mismo "un trozo de su propia realidad para que con ella se constituya el mundo". Ni acción que exprese un hacer, ni tampoco emanación. En rigor, se trata de creación. Es decir, propiamente es "una acción que pone una realidad transcendente a Dios, quien ejecuta el acto creador". Es una creación ex nihilo sui porque antes no existía. Pero además es una creación ex nihilo subjecti, pues todas las acciones que el ser humano realiza con carácter creador se realizan sobre un sujeto previo (PTHC 153).

Ahora bien, Dios no solo crea desde sí mismo sin emanación ni alteración ninguna, sino que además produce una alteridad, "para la cual no hay ningún

2. Por la extensión del tema de la creación, presentamos este artículo en dos números distintos de la Revista Latinoamericana de Teología. En el primero se conceptúa la creación; es el que exponemos a continuación. Y en el segundo se muestran los distintos modos de creación por parte de Dios; es la sección que queda pendiente por presentar. 
supuesto real previo fuera de la propia acción de Dios". Aquí es donde Zubiri se atreve a definir formalmente la creación como una acción que constituye una alteridad $\sin$ alteración ${ }^{3}$. En efecto, "la creación de un mundo transcendente a Dios significa que una realidad (una alteridad) es puesta sin alteración ninguna por parte de la realidad que lo ejecuta [...] ni por parte del término ejecutado" (PTHC 153)

La creación tiene dos momentos: por el primero, el término del acto creador es la realidad en cuanto realidad. Y de esta realidad se afirma que "es otra que la realidad de Dios". Por el segundo momento, esta alteridad no está hecha comenzando de ningún punto de partida (PTHC 153). Además, esta acción no implica ninguna alteración por parte de Dios. Pues bien, esta "alteridad de lo real sin alteración" es lo que aquí se llama una producción de lo real en cuanto real; justo en lo que consiste la creación (PTHC 154).

Definida la creación como una acción que constituye una alteridad sin alteración, se procede a continuación al análisis más pormenorizado del problema de la creación. Y se hace a partir del carácter formal del acto creador y de la estructura del acto creador de Dios.

\subsection{Carácter formal del acto creador de Dios}

Ante todo, cabe destacar que para Zubiri, Dios es una realidad absolutamente absoluta; en el sentido más intrínseco y pleno de la palabra, Dios es la realidad ${ }^{5}$. Esto significa que la transcendencia de su término es la alteridad de lo real en cuanto real. Es lo que propiamente constituye la creación. Es justo la posición de la alteridad de lo real en cuanto real, "sin alteración ninguna de la realidad que lo pone". Es lo que comúnmente se expresa cuando se afirma que Dios hace las cosas "desde la nada" (PTHC 154). Pero lo que con ello propiamente quiere decirse es que "no hay un sujeto anterior" (PTHC 155).

Pues bien, con la perspectiva de la creación "desde la nada", el horizonte del pensamiento ha sido trastocado totalmente ${ }^{6}$. Antes, el horizonte de pensamiento

3. Estas cursivas son nuestras.

4. Un análisis de estos dos párrafos de la página 153 de PTHC, en J. A. Navarrete Cano, "Materiales para la elaboración de una teología de la creación desde Zubiri. Análisis de un párrafo del libro el "Problema teologal del Hombre: Cristianismo" (sic), pp. 1-8.

5. Sobre la conceptuación de Dios como realidad absolutamente absoluta, véase nuestro trabajo en H. C. Gudiel García, La fe según Xavier Zubiri, pp. 112-115.

6. "La creación desde la nada no es, para Zubiri, un nuevo concepto que enriquece el elenco de los anteriores. Se trata, más radicalmente, de un concepto que cambia el horizonte mismo del pensamiento, de tal manera que todos los conceptos griegos, y también el concepto mismo de persona, van a ser situados en un nuevo sistema de 
era el de la movilidad. Ahora, en cambio, emerge un horizonte completamente nuevo ${ }^{7}$. En efecto, al horizonte del movimiento que constituye la razón griega, se yuxtapone ahora el "horizonte de la nihilidad, el horizonte de la creación y de la nihilidad" no-ser, y que las cosas son "entes". En consecuencia, Dios, que hace las cosas, es el Ser subsistente (PTHC 155).

Con estas tres observaciones falsas hemos llegado a lo que críticamente Zubiri llama la "entificación de la realidad" (PTHC 155) . Esta entificación se entendió como un nihilum de ser, y no como un nihilum de realidad. Es decir, se identificó el ser con la realidad; esta ha sido de hecho la perspectiva en la que ha nacido y se ha movido sucesivamente toda la metafísica occidental. Pero no se puede entificar la realidad ni por parte de la realidad finita, ni por parte de Dios, porque la realidad antes de ser es real. Y es que Dios no tiene ser, él está "allende el ser". Desde esta perspectiva, la creación es algo concreto. Por eso, no hay que distraerse "por aquello que se dice cuando nos referimos a Dios como creador" (PTHC 156).

En las Sagradas Escrituras existen dos fuentes o relatos de la creación, debidos a dos autores distintos. El más antiguo es el relato yahvista (cfr. Gn 2, 4b y ss.), escrito hacia el siglo $\mathrm{X}$ antes de Cristo. El segundo relato es el de Génesis 1, escrito por el así llamado código sacerdotal, aproximadamente en el siglo VI. Obviamente, se trata de dos fuentes que un solo redactor ha "enchufado" en un relato más o menos continuado, lo cual implica un problema más grave. Y es que en el primer relato se muestra a Dios como hacedor, mientras que en el segundo aparece Dios como creador $^{10}$. En tiempo de la monarquía, aparece Yahveh como un Dios único, creador de todo. Pero solo en tiempo de los macabeos,

referencia. Mientras que los griegos se han preguntado por las cosas reales desde el punto de vista de su movilidad, el hombre europeo, desde Agustín hasta Hegel, considerará las cosas reales desde el punto de vista de su nihilidad"; en A. González, La novedad teológica de la filosofía de Zubiri, p. 7.

7. Sobre la novedad de este nuevo horizonte, véase Sobre el problema de la filosofía y otros escritos (1932-1944), pp. 49-124; de ahora en adelante, la sigla de esta obra es SPFOE. También, de F. Aquino Júnior, "El carácter práxico de la teología. Un enfoque epistemológico", Revista Latinoamericana de Teología 82 (2011), pp. 101-120.

8. La importancia de la noción de horizonte puede verse en SPFOE 62-63.

9. La crítica madura que hace Zubiri a la entificación de la realidad va muy ligada a la crítica de la logificación de la inteligencia ( $c f r$. IRE 224-226). A mi juicio, esto trae significativas consecuencias para una correcta comprensión de la realidad, y para una adecuada interpretación de la misma inteligencia humana. La primera nos lleva a una desentificación de la realidad, y la segunda a una deslogificación de la inteligencia. Es una tarea crítica y necesaria.

10. Es lo que Zubiri expuso en la historia del monoteísmo; cfr. PFHR 222-224 (cfr. PTHC 157, nota 1). 
Dios aparece concretamente como un hacedor desde la nada $(2 \operatorname{Mac} 7,28)^{11}$ (cfr. PTHC 157).

Veamos a continuación, y más detalladamente, los pasos sucesivos de cada uno de los relatos de la creación.

\subsubsection{El relato yahvista: Dios hacedor}

El relato bíblico del yahvista presenta a Dios como hacedor de todo. Ahí se comienza con una frase temporal: "Cuando Yahveh Elohim creó los cielos y la tierra"12. Es la expresión usada para referirse a todo, es decir, a la "totalidad de lo real", tal como se conocía en la época. Expresar, pues, "los cielos y la tierra" es el modo común para decir "todo cuanto hay". Aquí aparecen los nombres de Yahveh y de Yahveh Elohim, y se yuxtapone el nombre de Elohim con el de Yahveh. De Yahveh Elohim se dice que es hacedor, hacedor de todo (PTHC 158).

Además, Dios hace el mundo, y cuando este fue creado, "no había yerba porque no llovía" ( $c f r$. Gn 2, 5). Era la sequía radical. Pero además de la falta de yerba, no hay ser humano tal que cultive el campo. De ahí la necesidad ineludible de que, "a la producción de algo que es totalmente inviable, sucedan distintos actos de creación que lo hagan viable". Esos actos de creación son los siguientes cuatro (PTHC 159).

Lo primero que Yahveh Elohim hace es al ser humano, dotado no de "cuerpo y alma", sino propiamente de "cuerpo y vida"13. En un segundo acto, este Dios "crea un territorio acotado y cerrado"; en hebreo, se llama Edén ( $c f r$. Gn 2, 8) $)^{14}$. Es la concepción imaginaria que tienen estos hombres del estado paradisíaco. El tercer acto muestra que este paraíso está lleno de árboles y agua. Y salen como fuentes cuatro ríos y unos árboles, etc. El hombre pone nombre a estas realidades por deseo de Dios: aquí el nombre significa apoderarse de la "naturaleza de las cosas". Finalmente, y ante la soledad del hombre, tenemos el sueño de Adán y la creación de Eva de una costilla (PTHC 159).

De este modo, la creación aparece como la "producción por parte de Dios de una cosa que al principio es inviable y que, por intervenciones ulteriores, se va haciendo viable gracias a la presencia del hombre y gracias a la presencia de

11. En 2 de Macabeos 7, 28, se lee: "Te ruego, hijo, que mires al cielo y a la tierra y, al ver todo lo que hay en ellos, sepas que a partir de la nada lo hizo Dios y que también el género humano ha llegado así a la existencia"; $N B J$.

12. "El día en que hizo Yahvé Dios la tierra y el cielo" (Gn 2, 4); NBJ.

13. Sobre esta precisión, véase también G. von Rad, El libro del Génesis, p. 92.

14. "Luego plantó Yahvé Dios un jardín en Edén, al oriente, donde colocó al hombre que había formado" (Gn 2, 8); NBJ. Sobre Génesis 2, 8, véase también G. von Rad, El libro del Génesis, p. 93. 
la humedad y a la fertilidad de la tierra". Antes de seguir con el texto, veamos a continuación el relato sacerdotal (PTHC 160).

\subsubsection{El relato sacerdotal: Dios creador}

El relato presentado por el sacerdotal expresa igualmente que "en el principio, creó Dios los cielos y la tierra" (Gn 1, 1). También aquí aparece la expresión el todo, tal como sucede en el relato yahvista. Sin embargo, el verbo hebreo utilizado aquí por el sacerdotal no es el 'aśah (hacer) del yahvista, sino el bara': es un hacer, pero en el sentido preciso de "crear". Y que en el Antiguo Testamento se aplica exclusivamente a Dios. Esto implica una evolución en el concepto de Dios y, sobre todo, en el concepto de creación porque ya no se trata de un simple hacer. El yahvista expresa que Dios, "como un alfarero, ha modelado al hombre "de la arcilla de la tierra y le insufló el aliento de vida"” (Gn 2, 7). En cambio, para el sacerdotal no se trata de un alfarero que con arcilla modela una figura, sino más bien "de un Dios que, con una sola palabra, produce aquello que dice". Por tanto, tenemos no solo a un Dios hacedor, sino además a un Dios “creador” (PTHC 160).

Lo primero que crea este Dios es una realidad caótica. Es lo que dice el libro del Génesis: "La tierra era caos y confusión y oscuridad por encima del abismo, y el espíritu de Dios aleteaba por encima de las aguas" (Gn 1, 2) (PTHC 160).

Este caos se expresa en tres conceptos sucesivos de gran importancia. Ante todo, está el concepto de jaleo, es decir el de caos, que es una especie de polvo, de torbellino. A esto le llaman en hebreo tohu wabohu, expresión que en francés todavía sobrevive como le tohu-bohu. Además, ese caos aparece con la "idea de las tinieblas: la indiferenciación de nada que sea una realidad concreta y determinada". Finalmente, el caos expresa cómo "el espíritu [ruaj] de Dios aleteaba [rajaf] sobre las aguas" ${ }^{15}$. El verbo rajab significa moverse (PTHC 161). Pero además puede pensarse que ruaj significa simplemente "el viento de Dios, la gran tormenta"16. En este caso, "el caos estaría expresado por la gran tormenta que pone en conmoción las aguas y se suma al torbellino del polvo del tohu-bohu y a las tinieblas" (PTHC 162).

Ahora bien, cuando el relato sacerdotal asume estas nociones, no implica que con ello esté haciendo un sencillo acto de sincretismo ${ }^{17}$. Porque aunque son nociones que la fuente sacerdotal toma de la civilización mesopotámica, las toma, pero no simplemente para sumarlas, sino sobre todo para otorgarles un nuevo sentido desde su concepción de Dios. Esto no es sincretismo. En rigor, es

15. "La tierra era caos y confusión y oscuridad por encima del abismo, y un viento de Dios aleteaba por encima de las aguas" (Gn 1,2); NBJ.

16. Sobre este significado de ruaj, véase Y. Congar, El Espíritu Santo, p. 30.

17. Una síntesis de la crítica de Zubiri al sincretismo puede verse en nuestra tesis H. C. Gudiel García, La fe según Xavier Zubiri, pp. 242-243. 
el modo "como se fuerza a la idea de la realidad de Dios que tiene el hombre a expandirse en todas las ricas posibilidades que constituyen su plenitud. Aparece Dios como creador del cosmos, en lo que tiene precisamente de caos material y de tierra, en lo que tiene de tiniebla y en lo que tiene de aguas" (PTHC 162).

Pero la creación tiene que proseguir para que deje de ser caótica, y se convierta en lo que los griegos llaman $\varkappa$ ó $\mu$ o, es decir, en una cosa ordenada. Aquí es donde comienzan los días de la creación (cfr. PTHC 162). Esta intervención creadora para hacer del caos un cosmos se expresa en el texto hebreo con el verbo badal, que significa separar. Esta separación se debe simplemente a la palabra de Dios, dabar Elohim 18 . "Esta palabra de Dios tiene una consecuencia, $\mathrm{y}$ es tan eficaz, que aquello que hace es justamente lo que ha querido hacer". Y por eso el Génesis dice "que es bueno" (Gn 1, 4) (PTHC 163).

El tipo de separación al que se refiere el texto aparece en la "exposición de los siete días de la creación". En primer lugar, "se trata de una separación de las grandes regiones que componen el cosmos. Es la obra de los tres primeros días de la creación" (PTHC 163).

El primer día de la creación, Dios "separa la luz de las tinieblas". Frente al caos tenebroso, Dios interviene diciendo: "Haya luz, y hubo luz" (Gn 1, 3) ${ }^{19}$. Y vio Dios que la luz "era buena” (Gn 1,4). Y añade que "separó la luz de las tinieblas”. He ahí el verbo badal, separar (PTHC 163).

En el segundo día de la creación, y mediante la creación de una bóveda metálica, se han separado unas aguas por encima del firmamento. Unas aguas “son las que constituirán la lluvia y el origen de los ríos y de los océanos, y las otras aguas, que quedan justamente en el fondo" del precipicio (PTHC 163). A esta bóveda se le llamó firmamentum, porque es "algo que está firme y sólido". Por tanto, se "separan las aguas de los cielos y las aguas de la tierra" (cfr. Gn 1, 6-8) (PTHC 164) ${ }^{20}$.

En el tercer día de la creación se separan la tierra firme y las aguas marinas ${ }^{21}$. Son las aguas que componen los mares y los océanos. Y estos produjeron "los primeros árboles y la primera vegetación" ( $c f r$. Gn 1,9-13). Sin embargo, para los hebreos, "los vegetales no son seres vivos" (PTHC 164) 22.

18. Sobre el valor noético y dinámico del dabar de Dios, véase R. Latourelle, Teología de la revelación, pp. 29-30; ahí mismo, puede leerse la importante nota 26. La función reveladora de la palabra de Dios, en S. Pie-Ninot, Teología fundamental, pp. 240-242. Además, B. Corsani, "Palabra", en P. Rossano, G. Ravasi y A. Girlanda (eds.), Nuevo diccionario de teología bíblica, pp. 1371-1390.

19. Véase también G. von Rad, El libro del Génesis, pp. 60-61.

20. Sobre estos versículos, véase también ibid., p. 63.

21. Véase también ibid., pp. 63-65.

22. Véase, además, ibid., p. 64. 
En consecuencia, tenemos la conformación de tres regiones: la región etérea: la luz; la región del firmamento; y la región terrestre y marítima. Son las tres grandes zonas que integran "la regionalidad del cosmos y que son el término primero de la creación” (PTHC 164).

Sin embargo, la creación de Dios no elimina del todo el caos de la materia, ni elimina del todo las tinieblas. Al separar la luz de las tinieblas, Dios llamó "a las tinieblas noche y a la luz día" (cfr. Gn 1,4-5). Pero tampoco Dios anula ese "carácter tormentoso de las aguas" (PTHC 164). Por tanto, esa creación consiste en que "son precisamente las fuerzas y poderes anticósmicos, que están ahí subyacentes a la creación y que, ciertamente, están dominados por el Dios que los ha creado, pero cuya acción está permitida dentro de las medidas marcadas por el Dios creador" (PTHC 164-165).

Además de esas tres regiones del universo, la acción creadora de Dios también ha creado las cosas que habitan esas regiones. Y por eso, el día cuarto, Dios ha creado aquello que puebla la región de la luz: los astros, sobre todo el sol y la luna. El relato sacerdotal recoge y reasume estos datos de una civilización y religión astral, pero lo hace con la intención de señalar el "carácter creador y supremo de Dios". Efectivamente, es Dios quien "ha creado los astros para que nos den luz y no simplemente para que sean dioses a los cuales el hombre adore" (PTHC 165) ${ }^{23}$.

En el día quinto de la creación, Dios puebla la región de las aguas y del aire. En la región de las aguas aparecen los monstruos marinos y los peces menores. En la región del aire surgen los pájaros, y la pueblan (cfr. Gn 1, 20-23) (PTHC 165) ${ }^{24}$.

En el día sexto de la creación, hay una separación sobre la tierra. Por un lado, surgen los animales que caminan sobre la tierra. Y por otro lado, aparece el ser humano (PTHC 165).

En este contexto del día sexto, "dijo Dios: "hagamos al hombre a nuestra imagen y semejanza" (Gn 1, 26). Dios ha creado al hombre y a la mujer con la orden explícita de "creced y multiplicaos" $(\mathrm{Gn} 1,28)^{25}$. Aquí la fuente sacerdotal ha tenido en consideración las dos ideas que más atemorizaban a un israelita. Ante todo, la idea de la hierogamia, es decir, "el pensar que el mundo nace de la copulación de dioses y realidades humanas". El texto sacerdotal se opone a la hierogamia. Y con ello, el sacerdotal también rechaza la idea de la

23. Cfr. Génesis 1, 14-19. "Los astros son considerados por completo creaturas dependientes de la voluntad ordenadora y creativa de Dios"; en G. von Rad, El libro del Génesis, p. 65.

24. Véase también G. von Rad, El libro del Génesis, pp. 66-67.

25. Un comentario de Génesis 1, 26-28 puede verse en G. von Rad, El libro del Génesis, pp. 68-72. 
prostitución sagrada de las "santas", es decir, "la práctica religiosa que hace pervivir y perdurar la hierogamia como reproducción y reactualización de la creación" (PTHC 166).

En definitiva, estos son los dos relatos bíblicos de la creación: el yahvista y el sacerdotal; ambos son bien distintos entre sí, como se ha podido notar en la exposición. Además, son relatos con una distancia temporal de cuatro siglos. No obstante, también es cierto que pueden encontrarse entre ellos algunas coincidencias significativas. Es lo que veremos a continuación ( $c f r$. PTHC 166).

\subsubsection{Coincidencias entre el yahvista y el sacerdotal}

Lo que en definitiva quiere ponerse de manifiesto en el relato yahvista y en el sacerdotal se reduce a una sola realidad: la "transcendencia de Dios"; esta transcendencia puede expresarse en las siguientes tres dimensiones (PTHC 167).

En primer lugar, los dos relatos coinciden en referirse a la totalidad de lo real. Ambos comienzan efectivamente diciendo "Los cielos y la tierra'. Y la creación como principio de toda realidad es creación ex nihilo" (2 Mac 7, 28) (PTHC 167) ${ }^{26}$.

En segundo lugar, tanto el yahvista como el sacerdotal coinciden en que es una creación "allende el tiempo". El sacerdotal termina diciendo que "el séptimo día [Dios] descansó" (Gn 2, 2) 27. Aquí hay una referencia al "reposo sabático"28. Se trata efectivamente de los siete días que terminan en el sábado. En consecuencia, es un relato estricta y particularmente religioso. El pueblo de Israel "ve en la creación el primer acto de la vida de Dios sobre la realidad entera, que es justamente el haberla creado" (PTHC 167).

Por tal razón, el inicio de la historia en el texto sacerdotal difiere del inicio de la historia en el texto yahvista. En el segundo, la historia viene inmediatamente. "Es la expulsión del paraíso por el pecado original. Y es una historia determinada". En cambio, el texto sacerdotal concluye afirmando que "estas son las generaciones de los cielos y de la tierra, cuando Dios las creó” (Gn 2, 4). Aquí

26. En 2 Macabeos 7, 28, se lee: "Te recuerdo, hijo, que mires al cielo y a la tierra $\mathrm{y}$, al ver todo lo que hay en ellos, sepas que a partir de la nada lo hizo Dios y que también el género humano ha llegado así a la existencia" (cursiva nuestra). La nota a este versículo en la Biblia de Jerusalén expresa que se trata de la "primera afirmación explícita de la creación ex nihilo"; NBJ.

27. El versículo completo de Génesis 2, 2 dice: "Y dio por concluida Dios en el séptimo día la labor que había hecho; y cesó en el día séptimo toda labor que hiciera"; $N B J$.

28. Sobre el tema, véase la nota a Génesis 2, 3 de la Nueva Biblia de Jerusalén. 
aparece la palabra hebrea toledot ${ }^{29}$, generaciones, traducida razonablemente por los buenos orientalistas por génesis (PTHC 167) ${ }^{30}$.

Sin embargo, a juicio de Zubiri, el término génesis ofusca lo que en este caso se pretende subrayar, que es fundamentalmente la idea de las generaciones. Idea propia de la fuente sacerdotal y que luego aparece en el resto del Génesis: son las generaciones de Adán, de Noé, de Sem, de Ismael, etc. ( $c f r$. Gn 5, 1; 10, 1; 11, 10; $25,12)$. Es una historia que se va contando por generaciones muy concretas. Esto no significa que para el sacerdotal, Dios es el generador del mundo. El concepto de generación es más bien "el esquema mental con que el pensar fantástico de estos hombres ha concebido la idea de la causalidad"31. Justo lo que sucede en Génesis $2,4^{32}$. De modo rudimentario, pero certero, aquí se quiere expresar algo evidente y aprehensible: la transcendencia de Dios (PTHC 168).

Pues bien, la transcendencia de Dios, llámesele a Dios según el yahvista o el sacerdotal, ha producido, ante todo, las cosas "de la nada" (2 Mac 7, 28). En esa transcendencia está precisamente lo que constituye la transcendencia del mundo. Porque, además, esa realidad ha sido producida al principio: "En el comienzo, creó Dios los cielos y la tierra" (Gn 1, 1) (PTHC 168). Así, hay en Génesis 1, 1, "ese concepto del comienzo transcendente del tiempo" (PTHC 168-169). Transcendente por tres razones: ante todo, porque Dios produce las cosas reales, pero las produce sin apoyarse en ninguna realidad. Transcendente, además, porque Dios antecede a todo tiempo de la creación. Transcendente, sobre todo y finalmente, porque Dios positivamente produce por su sola "palabra". Es lo que veremos a continuación (PTHC 168).

En tercer lugar, Dios es un "principio dominante", y domina por el mero hecho de su palabra (dabar). Dios crea por su sola palabra: "Haya luz, y hubo luz" (Gn 1, 3) $)^{33}$. A esta palabra, los primeros apologetas, inspirándose en el Evangelio de san Juan, la llamaron Logos. Pero con tal expresión no se referían

29. Sobre este término hebreo, puede verse la nota de Génesis $2,4^{\text {a }}$ en la Nueva Biblia de Jerusalén.

30. Una opinión sobre la voz génesis se encuentra en G. Ravasi, "Génesis", en NDTB, pp. 686-695.

31. Sobre la idea de causalidad en Zubiri, véase PFHR 126-129 ( $c f r$. PTHC 168, nota 1). Además, puede verse el reciente artículo de M. A. Sánchez Carrión, "Hume y Zubiri: el principio de causalidad", Estudios 106, XI (2013), pp. 43-64.

32. "Esos fueron los orígenes del cielo y la tierra, cuando fueron creados" (Gn 2, 4); NBJ.

33. Para Zubiri, esta es la traducción exacta de Génesis 1, 3, y nota a continuación que aquí no aparece la expresión "que la luz sea". No aparece el verbo ser. Con ello se está evitando la "falsa entificación de la realidad: que la luz "sea" (PTHC 169). Véase también G. von Rad, El libro del Génesis, p. 61. 
en modo alguno al logos inmanente, sino, sobre todo, al logos proferido ${ }^{34}$. La creación por la palabra es una idea que está presente a lo largo de todo el Antiguo y del Nuevo Testamento ( $c f r$. Sal 33, 6; 148, 5; 2 Pe 3, 5; St 1, 18; Rm 4, 17) $)^{35}$. Según el Evangelio de san Juan, "todo fue creado por el Verbo" (cfr. Jn 1, 3) (PTHC 169).

En el Nuevo Testamento, la noción de creación está fundada "sobre la propia estructura trinitaria de Dios (cfr. 1 Co 8, 6; Col 1, 15-20; Ef 1, 4-5; Hb 1, 1-8; Jn 1, 1-8)"36. Aquí la palabra se refiere al logos proferido de algo que es la verdad real de Dios, la misma realidad del Hijo (PTHC 169).

Más adelante veremos cómo la creación de las cosas reales se da "del Padre por el Hijo en el Espíritu Santo”. En efecto, “el Padre es la realidad absoluta, la fuente de toda realidad. Pero lo que toda la realidad puede ser es, justamente en su verdad, aquello que constituye la realidad misma del Hijo, que es la Verdad. Y en el Espíritu Santo es la actualización de la identidad de aquello que es verdad con lo que es realidad" (PTHC 170).

Desde esta concepción trinitaria de la creación hay que comprender "al Dios cristiano en su relación con el mundo". Naturalmente, la vida trinitaria expuesta en el artículo anterior es lo más sustantivo que hay que expresar de Dios. Pero ese decir trinitario de Dios está revelado justo para fundamentar la vida del hombre y, en consecuencia, su mismo "conocimiento de Dios". Pues bien, de Dios solo conocemos lo que es, pero en cuanto creador (PTHC 170).

En definitiva, con esto queda expuesta la primera sección de la primera parte del artículo: el carácter formal del acto creador de Dios. Se ha presentado a partir del relato yahvista de Dios como hacedor y del relato sacerdotal que entiende a Dios como creador. A pesar de las divergencias en cada relato, existen algunas coincidencias importantes expresadas a partir del carácter transcendente de la realidad de Dios. A continuación, veremos la segunda sección de la primera parte: la estructura del acto creador.

34. Sobre los dos estadios del Verbo, el logos inmanente y el logos proferido, en Teófilo de Antioquía véase L. A. Ladaria, El Dios vivo y verdadero, p. 201.

35. "De hecho, la Palabra marca el inicio y el término de la Biblia. En efecto, la historia de la Palabra se inició en la mañana de la creación, cuando por siete veces se afirma: "Dijo Dios..." (Gn 1, 3.6.9.11.14.20.24), y es en la Palabra hecha hombre que llega a la plenitud de su significado Jesucristo: "La Palabra (que) se hizo hombre..." (Jn 1, 14), ya que "Dios había hablado a los padres, pero ahora nos ha hablado en la persona del Hijo" (He 1, 1s.), que por esto "su nombre es Palabra de Dios" (Ap 19, 13); Pié-Ninot, La teología fundamental. "Dar razón de la esperanza” (1 Pe 3, 15), edición nueva, p. 252.

36. Veamos un solo ejemplo: "Para nosotros, no hay más que un solo Dios, el Padre, del cual proceden todas las cosas y para el cual somos; y un solo Señor, Jesucristo, por quien son todas las cosas y nosotros por él" (1 Cor 8, 6); NBJ. 


\subsection{Estructura del acto creador de Dios}

Recordemos que crear es propiamente una "acción del Dios transcendente", según los tres sentidos expuestos. He aquí la creación en cuanto creación desde Dios. Sin embargo, es menester preguntarse a continuación: "¿Y realmente qué es lo que Dios ha querido crear? ¿Cuál es su carácter real?”. En modo sintético, puede decirse que el propósito de la creación y la voluntad formal de Dios "es la plasmación ad extra de su propia vida trinitaria". Es lo que se pretende justificar a continuación (PTHC 172).

En este contexto, cabe poner de manifiesto una de las interferencias que se cruza entre la filosofía griega y la revelación bíblica: la idea en virtud de la cual las cosas reales que hay son naturales y que, por otro lado, está lo sobrenatural. Sin embargo, este concepto de lo sobrenatural es incorrecto, para Zubiri. Por eso, se pregunta con todo derecho, a continuación: “¿No será más bien que lo natural es una especie de concreción de lo sobrenatural?". De ser así, no habría sobrenaturalidad, sino más bien "la manera finita de tener vida divina sin ser Dios". Lo que aquí se define como naturaleza es tan solo "la finitud esencial con que la vida trinitaria se realiza ad extra". Lo sobrenatural no es una cosa sobrevenida; no se me da una naturaleza y, encima, se me da una cosa sobrenatural, gratuita. Pues bien, la creación "sería la plasmación de su propia vida trinitaria" (PTHC 173).

En ese caso se revela que la Trinidad como realidad procesional da de sí, en primer lugar, una esencia. El Padre como realidad absolutamente absoluta tiene una esencia abierta, y en la perijóresis "personal de las tres personas se constituye la propia esencia", la esencia divina (PTHC 173). El qué de Dios en cuanto acto puro es una consecuencia de "la vida trinitaria y no un principio de ella" (PTHC 174).

En segundo lugar, y además de las procesiones trinitarias, en Dios como realidad esencial constitutivamente activa, también está la "procesión de producir la realidad, es decir, su propia Trinidad, su propia vida trinitaria ad extra, como distinta de Dios". En rigor, aquí también se trata de una estricta procesión. En Dios también convergen las procesiones intratrinitarias y la "procesión ad extra. Es la plasmación ad extra de su propia vida trinitaria". Siendo así, "el mundo y toda la transcendencia del mundo consiste justamente en ser el precipitado transcendente de una procesión inmanente y vital, porque inmanente y vital es la procesión de la creación" (PTHC 174).

Esto complica las cosas, y por eso da lugar a tres cuestiones que a continuación hay que responder: qué es esa procesión ad extra por parte de Dios; qué es esa procesión ad extra por razón terminativa; y, uniendo ambas dimensiones, en qué consiste la estructura interna y efectiva de la creación. Veamos las respuestas sucesivas a estos problemas ( $c f r$. PTHC 174). 


\subsubsection{Qué es la creación por parte de Dios}

La explicación de la creación por parte de Dios se expresa en los siguientes tres conceptos esenciales, cada uno fundado en el anterior ( $c f r$. PTHC 174).

Ante todo, la creación tiene un carácter procesional. En otras palabras, el acto creador es un acto formalmente vital de Dios, propio de "esa actividad en que la realidad de Dios como un dar de sí consiste: es la procesionalidad". Además, la acción creadora es extática porque pone fuera de Dios algo distinto a Dios. Finalmente, en ese éxtasis, es posible y se expresa un carácter interior de la realidad de Dios: su propia infinitud (PTHC 175).

He aquí los tres conceptos que definen la naturaleza del acto creador por parte de Dios. Expliquemos más detenidamente cada uno de esos tres momentos: la procesionalidad, el éxtasis y la infinitud ( $c f r$. PTHC 175).

\section{a) El carácter vital del acto creador}

Este primer carácter es un acto que, de alguna manera, pertenece a la actividad que es propia de Dios, la cual, en cuanto "realidad absoluta y plenaria, es formalmente procesional". El acto creador, pues, es una procesión, obviamente no intratrinitaria. Este es el comienzo del problema (PTHC 175).

En primer lugar, recordemos que el Padre es la fuente y el "principio de toda realidad trinitaria". Lo que el monoteísmo tiene de principio primero le viene en definitiva del Padre. La unidad de la Trinidad efectivamente viene del Padre. En el Hijo hay una segunda suidad que "es la verdad real de lo que es el Padre. Y en el Espíritu Santo la ratificación, por vía de identidad, entre lo que es esa verdad y lo que es la realidad. Es el Espíritu de la Verdad" (PTHC 175).

Esto es de fundamental importancia para el tema que aquí nos ocupa porque, en definitiva, Dios crea, pero lo hace siendo trino. En cuanto principio de la Trinidad, el Padre "es también principio radical y personal de todo lo que no es Dios mismo. Y, en ese sentido, la esencia divina, en tanto que poseída por el Padre como suidad suya y propia, es aquello a que hay que referir en última instancia la creación". Ahora bien, la verdad real del Padre es precisamente la persona del Hijo. En consecuencia, el Padre crea, pero lo hace propiamente por el Hijo. Es lo que expresan san Pablo y san Juan: todo ha sido creado por él, por el Verbo ( $c f r .1$ Cor 8, 6; Jn 1, 3) ${ }^{37}$. Por tanto, "la creación es obra del Padre por el Verbo" (PTHC 176).

37. Para nosotros, dice Pablo, "no hay más que un solo Dios, el Padre, del cual proceden todas las cosas y para el cual somos; y un solo Señor, Jesucristo, por quien son todas las cosas y nosotros por él" (1 Cor 8,6). Según el Evangelio de Juan, "todo se hizo por ella [la Palabra] y sin ella no se hizo nada" $(J n$ 1,3); NBJ. 
Sin embargo, no basta con la mediación del Verbo en la creación, es necesario poner en acción la creación. Tal acción radica en "aquello que constituye el acto puro en que la realidad divina consiste, a saber: el propio Espíritu Santo" (PTHC 176).

Desde esta perspectiva, pues, la creación es "una especie de movilización de la esencia divina para la procesión de criaturas ad extra del Padre por el Hijo en el Espíritu Santo" (PTHC 176-177). Esto pone de manifiesto la estructura propiamente trinitaria de la creación. Esta procesión ad extra tiene una gran similitud con lo que son las procesiones trinitarias ( $c f r$. PTHC 177).

Comparativamente, puede aclararse lo anterior afirmando que "así como en las procesiones trinitarias se constituye la esencia infinita de Dios, así también en la procesión creadora se constituye la esencia finita de las cosas". En conclusión, la "esencia divina no es aquello que son las cosas, sino que la propia finitud de la esencia de las cosas realiza ad extra, en una o en otra forma, una procesión en Dios". Ahora bien, ¿qué es esta procesión? (PTHC 177). Es la cuestión que toca esclarecer a continuación.

El primer punto a recordar es que esta procesión lo es de alteridad, y que "consiste en producir otra cosa que no es Dios". Esto, obviamente, no sucede en las procesiones trinitarias (PTHC 177).

La procesión de alteridad no es ni generante del Hijo por parte del Padre, ni espirante del Espíritu Santo por parte del Padre y el Hijo. La procesión de alteridad es algo que puede llamarse, en opinión de Zubiri, una "procesión iniciante". Iniciante efectivamente porque aunque no se engendra ni se espira, "se inicia realidad". En otras palabras, la creación es una "iniciativa" de Dios. El mismo libro del Génesis 1, 26 da pie para pensarlo: "Hagamos al hombre a imagen y semejanza nuestra". He aquí "la expresión misma del acto de la iniciativa de Dios". Por tanto, además de las dos procesiones inmanentes de la generación y la espiración, en Dios también "hay una procesión transcendente que es la iniciante" (PTHC 178).

En el segundo punto por considerar se afirma que la procesión iniciante es absolutamente esencial. Es decir, es absolutamente esencial a Dios el haber podido crear el mundo, aunque no lo hubiera creado. De lo contrario, no sería Dios. Con la misma irrefragabilidad "con que Dios tiene procesiones trinitarias tiene también capacidad creadora, póngala o no la ponga en juego". Además de esencial, la creación tiene un carácter especial (PTHC 178). Esta procesión es transcendente, pero como acto es inmanente. Es un acto propio de la vida de Dios, fundado "en lo que es él de una manera constitutiva por las procesiones trinitarias" (PTHC 178-179). En otras palabras, la procesión de alteridad, en cuanto iniciante, no es constitutiva, pero sí es "intrínseca y esencialmente consecutiva a la realidad misma de Dios" (PTHC 179). 
En segundo lugar, si la creación es una procesión de alteridad en cuanto iniciante, hay que preguntarse a continuación en qué consiste esta iniciativa divina. Para responder y explicar esta cuestión, veamos las siguientes cuatro dificultades.

La primera dificultad manifiesta y que merece la pena clarificar es que Dios nunca ni en ningún ámbito toma iniciativas. Pero, en cambio, hay que afirmar con decisión que Dios sí tiene iniciativas, cosa totalmente distinta. Efectivamente, Dios tiene iniciativas porque es "iniciador e iniciante". Se trata de una verdadera iniciativa divina que es "iniciante por aquello en que consiste, a saber: en ser actividad, en el dar de sí. Dios en tanto que dando de sí en esta procesión ad extra es justamente iniciante e iniciador" (PTHC 179).

La iniciativa divina consiste, pues, "en el carácter activo que como realidad absolutamente plenaria compete a Dios, a saber: en su intrínseco, total y plenario dar de sî". Por tanto, Dios tiene iniciativas, lo cual significa "ser iniciador e iniciante" (PTHC 180).

La segunda dificultad sobre en qué consiste la iniciativa divina pone de relieve algo mucho más problemático. Ciertamente, es esencial a Dios el poder crear, pero crear no es forzoso a su carácter esencial, porque él pudo no haber creado el mundo. En consecuencia, la iniciativa de Dios para poder crear es una iniciativa totalmente libre. Ahora bien, ¿cómo definir la libertad de Dios? (cfr. PTHC 180).

Ante todo, la libertad no es una mera elección, como algunos creen:

Entre diversas cosas que se pueden hacer, yo elijo una. Si Dios no tiene forzosamente que crear, pero forzosamente para crear tiene que elegir, el acto creador sería una especie de segundo acto añadido a aquellos actos o aquel acto en que consisten las procesiones divinas y la propia realidad de lo que Dios es.

Este modo de pensar en algunos teólogos es totalmente insostenible y quimérico. El acto creador no es un segundo acto respecto de lo que Dios es como acto puro y como actividad primaria y radical. Ahora bien, si no es un segundo acto, queda en pie todavía el problema de que Dios pudo no haber creado. ¿Qué significa esto? (PTHC 180). Por un lado, significa que la creación "no es algo necesario y que, como pudo no haber existido, es contingente" (PTHC 181).

La tercera cuestión sobre la iniciativa divina expresa otro estrato del problema: ¿cómo se unifican en Dios esta necesidad y esta contingencia? Porque, efectivamente, las procesiones trinitarias de la generación del Hijo y la espiración del Espíritu Santo son necesarias en Dios. Esto es inexorable en Dios. Ahora bien, es menester aclarar que la necesidad de estas procesiones trinitarias en Dios es algo que sobrepasa todo lo que nombramos "necesidad física y metafísica desde el punto de vista de las criaturas". En Dios, se trata de una necesidad de 
otro tipo, indudablemente algo difícil de comprender, como sucede con todo lo que se refiere a Dios (PTHC 181).

Por eso, hay que señalar con energía y claridad que el "concepto de necesidad metafísica acuñado por la dialéctica y la metafísica griega no subsume el carácter de necesidad con que Dios produce sus procesiones trinitarias". Obviamente, este concepto de necesidad en Dios es totalmente distinto al "concepto griego de necesidad". En consecuencia, y de modo analógico, "el concepto de contingencia no puede aplicarse tampoco al acto creador". Se puede aplicar, pero solo en modo negativo: "Dios pudo no haber creado el mundo". Pues bien, "el concepto metafísico de contingencia de la realidad no se aplica para nada al poder no crear propio del acto creador" (PTHC 181).

Y es que ambos conceptos llevan a algo previo a la necesidad y a la contingencia. Es preciso, por tanto, descubrir una idea superior a estos dos conceptos. Por eso, se torna urgente la decisión de pasar de "la necesidad y de la contingencia con que concebimos las realidades del mundo a algo que está allende la necesidad y la contingencia". En efecto, esa actividad interna de procesión en que Dios consiste es justo una "efusión. Es una actividad efusiva" (PTHC 182).

Ahora bien, "el amor efundente puede ser en cierto modo irremediable sin abdicar de ser amor, y produce las procesiones trinitarias". Sin embargo, este amor puede tener un carácter diferente, "y es que el amor infinito de Dios depone su fruición en algo que es mucho menos que él". En eso consiste precisamente "la libertad. El mismo acto que, por un lado, es efusión hacia las procesiones trinitarias es, por otro, libertad de la creación. Dios es un dar de sí que es efusivo". Y, justo por serlo, el acto de la creación no es algo distinto al acto con que Dios se ama, sino que "es el propio acto con que Dios se ama, en tanto en cuanto depone esa fruición en que el amor consiste en una realidad que es inferior al acto de efusión" (PTHC 182).

El acto creador de Dios "no es un segundo acto". En realidad, es pura y simplemente el "mismo acto de amor en que quiere lo que es él efundentemente, en forma de generación y de espiración en el Hijo y en el Espíritu Santo, y en que quiere aquello que no es él, en forma de una iniciativa que es muy superior al amor con que esa iniciativa es iniciante". Es precisamente lo que aquí se llama "don, donación". En consecuencia, "el mundo, como término creador de Dios, es una donación en libertad". Precisamente por eso, la actitud del ser humano "frente a la creación está unívocamente determinada: es justamente el correlato humano y antropológico de la donación, que es la oblación". La idea del inicio del reposo sabático aparecida en Génesis 2, 23 es la primera en manifestar la oblación. Pues bien, la realidad y el hombre tienen un carácter "oblacional y oblativo" (PTHC 183). 
En cuanto "iniciativo, el acto de Dios es libre, porque depone la efusión en que formalmente consiste en una realidad que es infinitamente inferior a la propia. O, por lo menos, notoriamente inferior a la propia realidad divina" (PTHC 183).

La cuarta dificultad de en qué consiste la iniciativa divina es "¿cómo se compadece [unifica] este carácter iniciante e iniciativo de Dios con la eterna inmutabilidad divina?" (PTHC 184).

Ante todo, para enfocar y encauzar adecuadamente esa cuestión, Zubiri propone eliminar la palabra "eterno". Esto porque lo eterno ha significado que desde toda la eternidad, desde siempre, "Dios ha querido o no ha querido crear el mundo, y que si lo ha creado en un momento, ha introducido en su duración un momento para producir la creación del mundo". Pero esta concepción temporal de lo eterno es totalmente falsa. Y lo es porque Dios no es eterno en ese sentido. Propiamente hablando, Dios es eternal. Todo cuanto en Dios acontece, acontece por "modo eternal". En este sentido, la eternidad es un concepto modal de lo que es la realidad de Dios. Eterno no es "lo que no tiene principio ni fin". Si Dios "no tiene principio ni fin es porque es eternal. Pero no es que sea eterno porque no tiene principio ni fin" (PTHC 184). Es el carácter modal del tiempo en Dios el que fundamenta al carácter temporal de Dios y no al revés.

En rigor, pues, Dios es eternal. "Y la realidad más temporal y más contingente de la creación es vivida por Dios eternalmente". Pues bien, "la iniciativa creadora es una iniciativa vivida al modo como es Dios, a saber: eternalmente" (PTHC 184). Por eso puede decirse que Dios conoce la realidad contingente eternalmente. "Y como la consecuencia de lo eternal es que no tenga principio ni fin, de una manera incomprensible habrá que decir que eso estaba en una o en otra forma siempre presente en la mente divina". La eternalidad de Dios no elimina el carácter temporal de la creación. Sea como fuere, "Dios vive la iniciativa creadora eternalmente" (PTHC 185).

En definitiva, por parte de Dios, "la creación es una procesión inmanente por donación liberal, que constituye precisamente la finitud de una esencia en que se plasma la vida trinitaria”. Este es el primer carácter de la creación, pero es necesario ver a continuación el carácter extático de la creación (PTHC 185).

\section{b) Carácter extático del acto creador}

El segundo carácter del acto creador de Dios procede de "aquello que las personas divinas movilizan para que haya creación" (PTHC 185). En otras palabras, Dios tiene que poner en movimiento su inteligencia y su voluntad. Naturalmente, la esencia divina "es ya una esencia abierta a su propio carácter de realidad". Ahí es donde radica esa "identidad numérica en que consiste de una manera fundada la vida trinitaria, las procesiones trinitarias en Dios. Es 
una esencia que está abierta". Esta apertura es lo que en definitiva se denomina "éxtasis: estar fuera de sî". Pues bien, "la esencia divina es extática". Es el segundo carácter del acto creador (PTHC 186).

Por tanto, "la esencia divina como esencia extática es esencia abierta". Abierta no solo a sus propias procesiones trinitarias, como ya vimos, sino también a la procesión de la creación. Aquello que constituye el carácter extático de la esencia divina no es su imitabilidad, sino algo mucho más radical: "es su intrínseca, su metafísica y teologal fecundidad" (PTHC 186).

"La fecundidad de la esencia divina es aquello en que consiste formalmente el éxtasis de la esencia abierta de Dios". Esta esencia abierta está, ante todo, abierta a su propia realidad: "es el carácter de las procesiones trinitarias" (PTHC 186). Pero también, en esa esencia, Dios "está abierto a toda la realidad, precisamente por su fecundidad". Y en la medida en que esta fecundidad tiene su fundamento en la Trinidad, se abre el camino para mostrar "que el término de la creación consiste en la plasmación ad extra de la propia vida trinitaria" (PTHC 187).

Este es el segundo carácter del acto creador: su carácter extático. Queda por ver finalmente la infinitud del acto creador.

\section{c) Carácter de infinitud de la creación}

Hemos visto cómo la procesión iniciante conduce a la creación. Pero esta esencia divina no conduciría a la creación si esta fecundidad no tuviese un tercer carácter formal radical: su propia infinitud. La infinitud de la esencia divina se expresa en los siguientes tres conceptos (cfr. PTHC 187).

En primer lugar, la infinitud es la omnipotencia de Dios. En efecto, "Dios puede hacer todo lo que quiere. Nada es real sino por Dios" (PTHC 187). Ahora bien, la omnipotencia de Dios no se refiere formal y primariamente a la "dicción y al ser o a la contradicción y al contra-ser, sino a la realidad y a la contrarealidad" (PTHC 188). La contradicción o incontradicción no es el carácter primario de la realidad de Dios ( $c f r$. PTHC 187). Omnipotencia, pues, es potencia de realidad (PTHC 189).

En segundo lugar, la infinitud de Dios no solo es omnipotente, sino también es omnisciente, porque Dios lo sabe todo ( $c f r$. PTHC 188).

Ahora bien, Dios es omnisciente, pero lo es a posteriori, y este a posteriori no atañe a Dios, sino a la realidad. Ciertamente, Dios no sabe en este momento que estoy hablando, pero en cambio Dios sí lo sabe "eternalmente". Es mi acto el que está vivido eternalmente por Dios. Por tanto, "omnisciencia es eternalidad de aquello que, por lo menos, no solamente es posible, sino que es real en forma $a$ posteriori" (PTHC 189). 
En tercer lugar, y finalmente, la esencia de Dios es infinita por su providencia. La idea de la providencia significa que Dios crea un mundo en el que las cosas también están “queridas por sí mismas en su última realidad". He aquí la idea de la providencia. Una providencia que está fundada justo "en que la realidad de las cosas fuera de Dios, es en una o en otra forma, la realización finita de la propia vida trinitaria de Dios" (PTHC 189).

En conclusión, esto es lo que habría que decir de la creación por parte de Dios. Por tanto, la creación por parte de Dios “es una acción vital, una procesión iniciante en forma de donación liberal, que emerge justamente de la esencia de Dios en tanto que extática y en tanto que infinita en sus tres dimensiones de omnipotente, omnisciente y omniprovidente" (PTHC 189-190).

Pero además de la creación por parte de Dios, hay que ver a continuación la creación por parte del mundo.

\subsubsection{Qué es la creación por parte del mundo}

Ante todo, recordemos que, por parte del mundo, la creación "es aquello en que se realiza y como se realiza la procesión iniciante de la Trinidad". Ahora bien, hay que preguntarse a continuación "cuál es el carácter que tiene la creación en esta realización”. Es la pregunta que enseguida hay que responder (PTHC 190).

\section{a) Realidad y sentido de la creación}

Indudablemente, "la realidad de las cosas del mundo es distinta de la de Dios: en eso consiste la creación, en poner la alteridad de lo real en tanto que real sin alteración". Pero las cosas no solo son nudas realidades, sino que por serlo, tienen el carácter de ser cosa-sentido. No toda cosa-real ha llegado a ser cosa-sentido para el ser humano. Pero es innegable que toda cosa-sentido está fundada en las propiedades que posee como cosa-realidad (PTHC 190).

Desde la perspectiva de nuestra cuestión, esto significa que el término del acto creador es, ante todo, "la nuda realidad de las cosas, querida por Dios real y efectivamente en tanto que realidad. Dios quiere las cosas sencillamente por amor a las cosas mismas, a su propia realidad" (PTHC 190).

Pero, además, la realidad sí tiene sentido para Dios. "Y es que la efusión creadora de Dios depone su fruición en una realidad, que como realidad, sin dejar de serlo y precisamente por serlo, es infinitamente menor que la efusión que encuentra en ella su propia fruición". Este remanente se muestra como "una especie de peso que tiene la realidad divina en medio y encima de toda creación”. A este peso, en hebreo se le llama kabod, traducido en griego por doxa y en latín por gloria. La realidad es cosa-sentido justo "en la medida en que es 
gloria de Dios" ${ }^{" 38}$. Pero aquí gloria no significa que algo sea muy glorioso, sino solo aquello que "puede significar para un padre muy modesto y humilde que su gloria sea la realidad de su hijo y nada más". La realidad del mundo en cuanto realidad es justo aquello en que en rigor consiste "la gloria de Dios" (PTHC 191).

\section{b) La condición de la realidad}

Recordemos que toda cosa-sentido está apoyada en los atributos que tienen las cosas como "nuda realidad". Y a la capacidad que tiene lo real para ser constituido en sentido se le llama "condición" (PTHC 191)

Pues bien, la realidad en cuanto realidad tiene "una condición respecto de Dios, que es justamente el poder ser gloria suya". Y a esta condición se le llama el "bien, la bondad". En el libro del Génesis aparece precisamente esta realidad, diciendo que "Dios vio que la luz era buena, que vio que el mar era bueno, etc.". En este contexto, bueno no tiene una connotación meramente moral, ni significa que algo esté bien hecho (PTHC 191). En la interpretación de Zubiri, bueno significa la condición que tiene lo real en cuanto real para ser efectiva y formalmente gloria (kabod) de Dios (PTHC 192).

\section{c) La respectividad de lo real y de Dios}

Además de la condición de la realidad, todo lo real que procede de Dios, en cuanto real, es "respectivo". Esto constituye lo que aquí se llama el mundo en cuanto mundo ${ }^{40}$. "Todas las realidades que puedan salir de Dios se encuentran, en tanto que realidades, referidas las unas a las otras, por lo menos en la mente divina". El carácter del mundo es un transcendental de la realidad. Transciende de "todas las condiciones particulares de las cosas y de todos los xóøuol" (PTHC 192).

Ahora bien, Dios tiene respectividad, pero la tiene libremente. La prueba de este carácter de respectividad en Dios se manifiesta en el acto de redención. Dios tiene respectividad a la muerte redentora de Cristo en la cruz. Es respectividad real (cfr. PTHC 192).

Podrá ser libre tratándose de las existencias creadas por Dios. Y podrá ser simplemente "consecutiva en el orden de la fecundidad posible de la esencia divina". Pero esta respectividad, en uno u otro modo, compete a Dios en cuanto Dios. No obstante, se trata de una respectividad consecutiva y no propiamente

38. Véase H. U. von Balthasar, Gloria VI. Antiguo Testamento, pp. 31-36.

39. Es obvio que "con manteca no se puede hacer una puerta: evidentemente, las propiedades reales de la manteca no tienen condiciones para convertirse en puerta". Véase además EDR 228-229 (PTHC 191, nota 1).

40. Cfr. SE 428-432 (PTHC 192, nota 1). 
constitutiva a la realidad divina. Gracias a esto, "Dios, que produce la respectividad, no tiene sin embargo formalmente actualidad en ella; la realidad divina no es en sí misma respectiva". En consecuencia, "Dios, causa de la respectividad, al no tener actualidad en ella, carece de ser. Dios, causa de la respectividad, es causa de ese acto ulterior de la realidad creada que es el ser. Pero en sí mismo Dios está allende el ser" (PTHC 193).

\section{d) Carácter del mundo y la respectividad}

Ahora bien, este mundo y esta respectividad, incluyendo el cosmos, tienen un carácter muy particular: el mundo que Dios ha creado es un mundo abierto. Pero esta apertura no se refiere en modo alguno a que el mundo vaya formándose, porque evidentemente es un hecho de experiencia su formación. La apertura del mundo aquí se refiere más bien a que "es un mundo abierto por parte de Dios mismo". ¿Qué quiere decir esto? (PTHC 193).

Si recordamos los dos relatos bíblicos expuestos, podemos ver que, en forma distinta, pero innegable, "Dios va produciendo creaciones sucesivas" (PTHC 193). Desde el relato yahvista de la creación, "Dios primero hace al hombre, después hace las aguas y la lluvia, después hace a la mujer, etc.". En la perspectiva del relato sacerdotal, "después de la creación del caos, va en seis días creando sucesivamente el mundo" (PTHC 194).

La interpretación de Zubiri sobre esta "sucesividad" de la creación expresa que las "distintas intervenciones de Dios son real y efectivamente distintas intervenciones". Y que, en consecuencia, "en la primera no estaban formalmente incluidos lo que iba a ser la segunda y lo que iba a ser la tercera". En este sentido, "las iniciativas de Dios son iniciativas sucesivas. De lo contrario, no pareciera sino que Dios no ha tenido más que una iniciativa de crear el mundo: dijo fiat y se acabó. No ha asistido más que a la realización de su decreto" (PTHC 194).

En realidad, Dios se ha reservado "sus iniciativas para ir agregando, iniciativa tras iniciativa, el detalle al mundo que efectivamente va a salir de sus manos". Esto es fundamental (PTHC 195).

Lo antes expuesto se refiere a las distintas "posibilidades de interpretación que el Génesis deja abiertas y al mundo creado por Dios, tomado con todos sus detalles no solo materiales, sino también humanos". A este mundo así considerado se le llama mundo abierto. La enorme cantidad de "sucesivas iniciativas divinas es esencial tratándose de la vida humana. Solo si incluimos en el mundo estos detalles de la vida humana puede hablarse de un mundo abierto" (PTHC 195).

Obviamente, "cada una de estas iniciativas divinas está eternalmente vivida. En este sentido, todo es simultáneo en Dios". Por eso, el mundo "es un mundo abierto a las propias iniciativas divinas, porque Dios no se ha contentado con tomar una iniciativa en la que ha agotado los detalles del mundo" (PTHC 195). 
Este carácter abierto de las iniciativas divinas califica a este mundo con los siguientes dos conceptos, cada uno fundado en el anterior. Ante todo y formalmente, el mundo que Dios ha creado en su primer fiat es "el teatro de sus propias iniciativas ulteriores. Este teatro es la realidad material entera. Dios no ha agotado sus iniciativas con el primer fiat creador". Además, y en profunda unidad y dependencia con lo anterior, los términos producidos por las iniciativas anteriores son "el substrato sobre el cual están montadas las ulteriores iniciativas divinas" (PTHC 196).

Pero además de teatro y substrato de las iniciativas divinas, "tomando el mundo entero de las iniciativas divinas, este mundo abierto que se va conformando es pura y simplemente el devenir teologal de las propias iniciativas; es decir, de la acción iniciante con que ad extra Dios constituye las cosas". Obviamente, Dios no deviene en sí mismo, pero sin duda deviene en otro: en el mundo. Y este devenir en el otro es justo la vinculación "sistemática y progresiva, la concatenación teologal de sus propias iniciativas divinas" (PTHC 196).

\subsubsection{La realidad integral de la creación}

Si ahora unificamos "lo que es la acción creadora por parte de Dios y lo que es el mundo creado para gloria suya y abierto, podemos decir que en la creación, Dios nos aparece como la realidad fontanal" (PTHC 196-197) ${ }^{41}$.

Esta realidad fontanal tiene un carácter particular. En efecto, Dios "ha querido que estas realidades sean lo más divinamente reales que cabe, es decir, que se vayan formando a sí mismas. Es la voluntad de autoformación con que Dios ha querido un mundo que se va formando y que, además, va a hacer que se vaya formando en buena parte por iniciativas distintas. La voluntad de creación es una voluntad formalmente autoformativa". En esa medida, el mundo es una imagen "de lo que es la propia existencia de la vida divina" (PTHC 197).

Ahora bien, con lo anterior no quiere decirse que el mundo tenga un vestigio de la Trinidad, como pensaron los teólogos neoplatonizantes. En realidad, se trata positivamente de que "el carácter trinitario de la acción creadora es el que plasma y ha querido plasmar efectivamente un mundo [...] que es justamente una autoformación en la que intervienen en buena medida [...] las distintas iniciativas divinas. Dios es fontanal y además naturante de este mundo abierto. Y este mundo no es nunca ajeno a la realidad y a la acción de Dios" (PTHC 197).

No se trata solo de "que haya realidades, sino que Dios ha querido que las realidades se hagan reales de una manera lo más divinamente posible, a saber: por sí mismas" (PTHC 198). Y por esto, la creación es un dar de sí "como donación de Dios, no solamente en el orden de dar la realidad, sino además de dársela

41. Sobre el concepto de fontanalidad divina, véase HD 177-178 ( $c f r$. PTHC 197, nota 1). 
lo más divinamente posible, a saber: que con un substratum primario, subyacente al cual está fontanalmente Dios, las cosas vayan haciéndose a sí mismas de una manera mediata" (PTHC 199).

Pero con lo antes expuesto en esta primera sección del trabajo, en la que hemos respondido a la cuestión de qué es la creación, tan solo hemos entrado en el tema de la creación. Porque, efectivamente, hay diversas maneras y diversos tipos de creación. "¿En qué consisten y cuáles son?”. En ellos se entra en la concepción de la realidad del hombre como persona creada. Es justo la cuestión de la "antropología teológica", tema que abordaremos a continuación (PTHC 199). (Continuará.)

\section{Siglas usadas para la obra de Zubiri}

EDR: Estructura dinámica de la realidad, Madrid, 1989.

IRE: Inteligencia sentiente. Inteligencia y realidad, Madrid, 1991

PFHR: El problema filosófico de la historia de las religiones, Madrid, 1994²re.

PTHC: El problema teologal del hombre: Cristianismo, Madrid, 1997.

SE: Sobre la esencia, Madrid, 1985.

SH: Sobre el hombre, Madrid, 1998.

SPFOE: Sobre el problema de la filosofía y otros escritos (1932-1944), Madrid, 2002. 